\title{
Violência Doméstica e Crenças: Intervençáo com Profissionais da Atenção Primária à Saúde*
}

\author{
Domestic Violence and Beliefs: Intervention with Health Primary Care Professionals
}

\author{
Camila Resende Soares Brum ${ }^{1}$ \\ Lélio Moura Lourenço ${ }^{\text {II }}$ \\ Carla Ferreira de Paula Gebara ${ }^{\text {III }}$ \\ Telmo Motta Ronzani ${ }^{\mathrm{II}}$
}

\begin{abstract}
Resumo
A presente pesquisa teve como objetivo principal investigar as crenças e atitudes dos profissionais da Atenção Primária à Saúde em relação à violência doméstica. Participaram da pesquisa 65 profissionais que responderam a um questionário autoaplicável, estruturado, individual e anônimo. O instrumento foi aplicado antes e após uma capacitação conceitual sobre violência doméstica, com o intuito de analisar as crenças e as possíveis mudanças a partir deste tipo de intervençáo. Os dados foram analisados por meio do programa SPSS e pelo teste de significância McNemar. Os resultados indicaram mudanças de crenças significativas, a partir da intervenção proposta quanto à forma do profissional do Programa Saúde da Família em lidar com vítimas de violência doméstica; ao papel do trabalhador da atenção primária em detectar pacientes com problemas relacionados à violência doméstica, ao interesse profissional sobre o padrão, os riscos de saúde relacionados e o aconselhamento para diminuir ou pará-la. Os dados não indicaram mudanças referentes à confiança que os profissionais sentem em sua habilidade para lidar com a questão.
\end{abstract}

Palavras-chave: Violência doméstica; profissionais de saúde; crenças; prevenção.

\section{Abstract}

This research focused on investigating the Primary Health Care providers' beliefs and attitudes towards family violence issues. The research involved 65 professionals who responded a self-administered, structured, and anonymous questionnaire. In order to examine possible changes that resulted from this type of intervention, the instrument was administered before and after a conceptual training on family violence. Data was analyzed using the SPSS program, and McNemar's significance test. The results, from the proposed intervention, showed some significant changes in the Primary Health Care providers' beliefs on how to deal with victims of family violence, as well as in the role the Primary Health Care provider plays when detecting patients with family violence-related problems; and their relation to the professional standards, health risks, and counseling skills used to decrease or stop it. The data did not indicate changes in the level of confidence that Primary Health Care providers have in their ability to deal with the issue.

Keywords: Domestic violence; health professionals; beliefs; prevention.

${ }^{\mathrm{I}}$ Centro de Prevenção à Criminalidade de Juiz de Fora

"Universidade Federal de Juiz de Fora

IIIUniversidade Federal de São Paulo

A questão da violência doméstica, que ocorre na esfera das relaçóes interpessoais, vem ganhando destaque no meio acadêmico, tanto pela magnitude, como pelas repercussóes do problema para a saúde dos indivíduos e das coletividades (Gebara, Cezario, Ronzani \& Lourenço, 2010). A Organizaçáo Mundial de Saúde (2002) enfatiza a necessidade de reconhecer a imensa parte invisível da violência que não resulta necessariamente em mortes ou lesóes graves, mas oprime e gera danos físicos, psicológicos e sociais nos indivíduos que se encontram submetidos de forma crônica aos abusos. É o caso das violências domésticas e intrafamiliares, com agressóes físicas, sexuais e psicológicas, além da privação e negligência, que acometem, sobretudo, mulheres, crianças e idosos.

Um recente estudo bibliométrico (Bhona, Lourenço \& Brum, 2011) sobre violência doméstica indicou que a mulher destaca-se como a principal vítima entre as publicaçóes analisadas no período de 2006 a 2009. Estima-se que uma a cada cinco mulheres sofrem alguma forma de violência durante a vida, podendo levar à lesão grave ou morte. Além disso, em cerca de um quarto dos casos, o abuso sexual também ocorre (UN Millennium Project, 2005). Dados da Pesquisa Nacional por Amostragem Domiciliar (PNAD) indicam que 63\% dos casos de agressão 
física ocorridos nos domicílios tiveram como vítima o sexo feminino (Brasil, 2002a). Os agressores, em sua grande maioria, são conhecidos, sendo identificados com maior frequência como maridos, companheiros ou parentes próximos. Estudos em todo o mundo apontam uma elevada prevalência e variabilidade da violência entre parceiros íntimos, entre 15 a $71 \%$ (Ellsberg, Jansen, Heise, Watts \& Garcia-Moreno, 2008; Garcia-Moreno, Jansen, Ellsberg, Heise \& Watts, 2006, Schraiber et al., 2007; Vieira, Perdona \& Santos, 2011).

Alguns aspectos destacam-se no que diz respeito às barreiras para a realização de açôes que promovam mudanças (Organização Mundial de Saúde, 2002). Nem sempre a vítima do dano percebe o ato como violento. Ainda, outro fator relevante é a postura dos profissionais diante dos casos de violência doméstica. $\mathrm{O}$ respeito à pessoa que sofreu violência nem sempre é observado nos diversos serviços existentes. É comum as mulheres serem responsabilizadas ou culpadas por isso. Essa conjuntura dificulta a tomada de atitudes por parte das mulheres, tanto para denunciar as agressóes, quanto para reagir de maneira efetiva modificando a situação vivida (Brasil, 2002b).

Nesse sentido, estudos e intervençôes que avaliem e busquem promover mudanças nas crenças e atitudes dos profissionais atuantes em situaçóes de violência doméstica tornam-se relevantes, na medida em que estas mudanças podem influenciar açóes adequadas para prevenção e promoção de saúde de vítimas e demais pessoas envolvidas.

Portanto, o Programa Saúde da Família (PSF) encontra-se em posiçáo privilegiada para atuação preventiva da violência doméstica, devido à proximidade com a comunidade local, à atuação intersetorial, ao estabelecimento de parcerias, ao diálogo constante com diferentes setores e, primordialmente, à responsabilidade na promoção da saúde.

No estudo da violência doméstica, uma análise das crenças dos profissionais que lidam diretamente com este fenômeno se torna importante (Gebara et al., 2010), já que elas se constroem a partir da experiência direta (Cavazza, 2008; Krüger, 1995). Partindo do pressuposto de que as crenças determinam o modo como o ser humano se posiciona e reage diante de situaçóes, e a forma como o profissional de saúde concebe a violência doméstica influencia o modo como o mesmo atuará frente ao problema (Sinclair, 2010), o presente estudo teve como objetivo avaliar as crenças e suas possíveis mudanças dos profissionais de saúde em relação à violência doméstica em serviços de Atenção Primária à Saúde, por meio de uma metodologia de intervenção voltada para a capacitação destes profissionais para atuarem de forma preventiva.

\section{Materiais e Métodos}

A presente pesquisa está inserida em um projeto do Polo de Pesquisas em Psicologia Social e Saúde Coletiva (POPSS), da Universidade Federal de Juiz de Fora, iniciado em 2009, intitulado "Avaliação de Processo sobre Prática de Prevenção ao Uso de Drogas e Violência Doméstica em Serviços de Atenção Primária à Saúde". A pesquisa ocorreu em quatro municípios de pequeno porte da Zona da Mata Mineira, com menos de 40.000 habitantes. A escolha dos mesmos justificou-se pelas características dos sistemas de saúde locais, organizados de forma horizontal, com uma maior proximidade entre gestores, profissionais e usuários dos serviços se comparados a municípios maiores, o que facilita a avaliaçáo e o acompanhamento das atividades.

Foram definidos os seguintes critérios de elegibilidade para os municípios participantes: aceite formal da Secretaria Municipal de Saúde em participar do projeto; capacitação das equipes de PSF completas; disponibilização de horas de trabalho dos profissionais para participarem da parte presencial da capacitação; maior número de profissionais inscritos; municípios com menos de 40.000 habitantes e ter PSF implantado no município.

Após contato com os gestores das Secretarias Municipais de Saúde, para autorização e apoio à efetivação do projeto, realizou-se uma sensibilização, por meio de reunióes com os setores: conselhos municipais de saúde, conselhos tutelares, delegacias, assistência social, comunidade civil organizada (AA), gestores e coordenadores de saúde e outros setores importantes para o processo. Num segundo momento, os profissionais selecionados para participarem deste estudo, assim como os gestores e facilitadores, passaram por uma capacitação sobre práticas de prevenção ao uso de substâncias psicoativas e violência doméstica, divididos em três módulos de aproximadamente quatro horas.

Quanto à violência doméstica, o treinamento buscou desenvolver competências conceituais, comunicativas, interpessoais e políticas dos profissionais em relação ao impacto da violência sobre a saúde; às ações de prevenção; ao atendimento das 
vítimas e ao gerenciamento de ações. O conteúdo da capacitação foi baseado em materiais de orientação para profissionais de saúde, elaborados e publicados pelo Ministério da Saúde (Brasil, 2002a,b). Foram apresentados e discutidos conceitos e crenças referentes à violência doméstica de uma forma geral, enfatizando o papel da mulher neste contexto, por ser esta a forma mais frequente de violência sofrida pelas mulheres. Além disso, estas são as principais usuárias de serviços de saúde, especialmente os da atenção primária (Schraiber et al., 2003), o que poderia favorecer a execução de açóes preventivas.

\section{Instrumentos}

Para a realização deste estudo foram utilizados dois instrumentos, sendo os mesmos aplicados antes e após a capacitação. Isso ocorreu antes da sensibilizaçáo em cada município, para que fosse possível avaliar as crenças dos profissionais antes da capacitação. Antes do uso, tendo em vista que muitos profissionais demonstraram dúvidas ou mesmo desconhecimento acerca do projeto, realizou-se primeiramente uma explanação sobre o mesmo e seu objetivo. Em seguida, os participantes assinaram o termo de consentimento livre e esclarecido devidamente aprovado pelo Comitê de Ética em Pesquisa da Universidade Federal de Juiz de Fora e receberam as instruçóes relativas ao preenchimento.

\section{Questionário sociodemográfico}

Houve um questionário sociodemográfico composto por 25 questóes, com o objetivo de caracterização da amostra. Destas, foram analisadas as variáveis: gênero, estado civil, composição familiar, escolaridade, profissão, local de trabalho e participação prévia em algum tipo de treinamento.

\section{Questionário sobre práticas de prevenção à violência doméstica}

O questionário é composto por uma primeira parte, que avalia as práticas de prevenção à violência doméstica e, uma segunda, que analisa as crenças dos profissionais quanto à tal situação. Este instrumento foi utilizado em pesquisas anteriores que avaliaram as crenças dos profissionais da atenção primária em relação à prevenção ao uso de substâncias psicoativas (Ronzani et al., 2008, 2009), e realizou-se uma adaptação livre do mesmo para a violência doméstica.
No presente estudo foram adaptadas e analisadas 36 questôes sobre atitudes e crenças dos profissionais com relação aos problemas devido à violência doméstica pelos seus pacientes, com respostas em uma escala do tipo Likert de cinco e quatro pontos. Foram apresentadas afirmaçóes sobre: como lidar com tais vítimas; o papel do profissional da atençáo primária em detectar pacientes com problemas relacionados à violência doméstica; $\mathrm{o}$ interesse profissional sobre o padrão da violência doméstica e os riscos de saúde relacionados a isso ou o aconselhamento para diminuir ou pará-la e o grau de confiança que o profissional tem na sua habilidade em desempenhar determinadas atividades. $\mathrm{O}$ participante era orientado a ler cada uma das questôes, assinalando numa escala de um a cinco, em que um correspondia a "discordo totalmente" e cinco a "concordo totalmente", ou um a quatro, na qual um correspondia a "sem confiançà" e quatro à "bastante confiança”, aquela que melhor descrevia como ele se sentia.

Em seguida, os participantes assinaram o termo de consentimento livre e esclarecido e receberam instruçôes relativas ao preenchimento.

\section{Análise de dados}

Os questionários foram digitados no software Statistical Package for the Social Science (SPSS, versão 15) para organização das variáveis e construção de um banco de dados. Possíveis erros de digitaçáo e inconsistência dos dados foram conferidos e corrigidos. Posteriormente, iniciaram-se as análises estatísticas descritivas pelo cálculo das frequências absoluta (f) e relativa (\%) para as variáveis categóricas.

Partiu-se para as análises inferenciais a fim de testar as hipóteses de que algumas crenças e atitudes dos profissionais de APS sofreriam mudanças após a capacitação proposta. Para tanto, foram comparadas as respostas de cada item do questionário sobre crenças e atitudes relativas à violência doméstica, aplicados antes e após a capacitaçáo. Os itens avaliados a partir de escalas do tipo Likert de cinco pontos, com respostas variando de "discordo totalmente" a "concordo totalmente", foram recategorizados em três pontos (discordância, neutro e concordância). Já aqueles por escalas do tipo Likert de quatro pontos, referentes à confiança dos profissionais em realizar açóes de prevenção em violência doméstica, que variavam de nenhuma à muita confiança, foram recategorizadas em dois pontos ("Nenhuma/muito pouca confiança" e "pouca/ muita confiança"). 
Assim, pretendeu-se observar se existia uma mudança de algum tipo de concordância para discordância, pois dessa forma os resultados possuem maior relevância prática a fim de direcionar uma futura capacitação. Mudanças tênues entre os níveis de discordância — "discordo totalmente" para "discordo parcialmente” - seriam insuficientes para avaliar a mudança efetiva de uma crença.

\section{Resultados}

Participaram do estudo 65 profissionais do PSF, em sua maioria do sexo feminino $(84,6 \%)$, sendo que $58,5 \%$ não possuíam cônjuge/companheiro e $60 \%$ não tinham filhos. Com relação à escolaridade, 53,8\% tinham concluído o ensino médio; $27,7 \%$, ensino técnico e $10,8 \%$, curso superior. No que tange à ocupação dos profissionais no PSF, predominaram os agentes comunitários de saúde (72,3\%), seguidos de técnicos ou auxiliares de enfermagem (13,8\%), enfermeiros $(6,2 \%)$, médicos $(3,1 \%)$, técnicos em saúde bucal $(3,1 \%)$ e dentistas $(1,5 \%)$.

\section{Como lidar com vítimas de violência doméstica}

Em relação a como lidar com vítimas de violência doméstica (Tabela 1), nove profissionais $(13,8 \%)$ afirmaram conhecer o suficiente sobre as causas dos problemas relacionados ao fenômeno antes da capacitaçáo, sendo que, após a mesma, $18(27,7 \%)$ passaram a concordar com esta afirmação $\left(\chi^{2}=9,46 ; p=0,02\right)$.

Quando questionados quanto ao aconselhamento, antes da capacitação 15 profissionais $(24,6 \%)$ mencionaram que podiam orientar apropriadamente seus pacientes sobre a violência doméstica e seus efeitos, sendo que após a capacitação 27 deles $(43,1 \%)$ passaram a concordar com tal afirmação $\left(\chi^{2}=12,69 ; \mathrm{p}=0,01\right)$.

Antes da capacitação, 15 sujeitos (24,6\%) concordaram com a afirmação de que não tinham muito a oferecer aos pacientes vítimas de violência doméstica e, após a mesma, nove $(13,8 \%)$ passaram a afirmar o mesmo. Destaca-se que os profissionais não passaram a discordar, mas passaram a ocupar um posicionamento neutro $\left(\chi^{2}=8,64 ; \mathrm{p}=0,04\right)$.

\section{Papel do profissional}

Em relação ao papel do profissional (Tabela 2), 11 respondentes $(17,2 \%)$ afirmaram estarem muito ocupados com outros problemas de saúde e, após a capacitação, 13 (20,3\%) passaram a concordar com esta afirmação. Apesar disso, após o treinamento, o número de profissionais que discordavam diminuiu — de $46(71,9 \%)$ para $30(46,9 \%)$ profissionais. Este dado indica que os mesmos passaram a se posicionar de forma neutra - antes da capacitaçáo sete $(10,9 \%)$ não concordavam, nem discordavam, ao passo que após este número passou para $21(32,8 \%)$, com $\chi^{2}=13,95 ; p=0,003$.

\section{Interesse}

Já sobre o interesse do profissional ao padrão de violência doméstica, aos riscos de saúde relacionados e ao aconselhamento (Tabela 3), no que diz respeito a não saber abordar efetivamente os pacientes para reduzir a violência doméstica, 31 profissionais $(47,7 \%)$ concordavam com a afirmação e, após a capacitação, este número passou para 20 (30,8\%). Houve um aumento daqueles que começaram a discordar da afirmação, bem como daqueles que se posicionaram de maneira neutra $\left(\chi^{2}=8,37 ; \mathrm{p}=0,04\right)$.

Antes da capacitação, 15 indivíduos (24,2\%) concordavam que não havia tempo suficiente para aconselhar os pacientes sobre a violência doméstica e após, 13 profissionais $(21,0 \%)$ concordaram com tal afirmação. Em contrapartida, houve decréscimo do número de profissionais que discordavam disso após a capacitaçáo - de 40 (64,5\%) para 32 (51,6\%). Ressaltase, portanto, a mudança ocorrida com relação a uma posição neutra, que foi de $7(11,3 \%)$ para $17(27,4 \%)$ profissionais após a capacitação $\left(\chi^{2}=7,68 ; \mathrm{p}=0,05\right)$.

\section{Confiança}

Já sobre a confiança que os profissionais de saúde sentem em sua habilidade para lidar com casos de violência doméstica, os resultados não apontaram mudanças significativas quando comparados os dados coletados antes e após a capacitação.

Assim, é possível destacar alguns dados que não dizem respeito ao impacto da capacitaçáo, mas como os profissionais se posicionaram em relação a este item, tendo como referência as respostas coletadas após a capacitação. Quando questionados a respeito de fazer perguntas de natureza pessoal ou delicadas ao entrevistar um paciente, $35(54,7 \%)$ profissionais disseram ter nenhuma ou pouca confiança, enquanto $29(45,3 \%)$ afirmaram ter pouca ou muita. Portanto, esta é aquela ação em que os profissionais demonstraram ter menos confiança 
Tabela 1. Como lidar com vitimas de violência doméstica.

E) Eu acho que conheço o suficiente sobre as causas dos problemas relacionados à violência doméstica para desempenhar meu papel ao lidar com as vítimas ( $\mathrm{n}=65)$.

\begin{tabular}{lccc}
\hline Respostas & $\begin{array}{c}\text { Antes } \\
\mathbf{n}(\mathbf{\%})\end{array}$ & $\begin{array}{c}\text { Depois } \\
\mathbf{n}(\%)\end{array}$ & Teste McNemar-Bowker \\
\hline Discordam & $37(57,0)$ & $24(36,9)$ & \\
Neutros & $19(29,2)$ & $23(35,4)$ & $\chi^{2}=9,46 ; \mathrm{p}=0,02$ \\
Concordam & $09(13,8)$ & $18(27,7)$ & \\
\hline
\end{tabular}

F) Eu acho que posso aconselhar apropriadamente meus pacientes

sobre a violência doméstica e seus efeitos $(\mathrm{n}=63)$.

\begin{tabular}{lccc}
\hline Respostas & $\begin{array}{c}\text { Antes } \\
\text { n (\%) }\end{array}$ & $\begin{array}{c}\text { Depois } \\
\text { n (\%) }\end{array}$ & Teste McNemar-Bowker \\
\hline Discordam & $24(37,7)$ & $13(20,0)$ & \\
Neutros & $24(37,7)$ & $23(36,9)$ & $\chi^{2}=12,69 ; \mathrm{p}=0,01$ \\
Concordam & $15(24,6)$ & $27(43,1)$ & \\
\hline
\end{tabular}

G) Eu acho que náo tenho muito a oferecer aos pacientes vítimas de violência doméstica (n=62).

\begin{tabular}{lccc}
\hline Respostas & $\begin{array}{c}\text { Antes } \\
\mathbf{n}(\%)\end{array}$ & $\begin{array}{c}\text { Depois } \\
\mathbf{n}(\%)\end{array}$ & Teste McNemar-Bowker \\
\hline Discordam & $27(43,1)$ & $20(32,3)$ & \\
Neutros & $20(32,3)$ & $33(53,9)$ & $\chi^{2}=8,64 ; \mathrm{p}=0,04$ \\
Concordam & $15(24,6)$ & $09(13,8)$ & \\
\hline
\end{tabular}

Tabela 2. Papel do profissional de atenção primária em detectar pacientes com problemas relacionados à violência doméstica.

\begin{tabular}{lccc}
\hline H) Os profissionais de atençáo primária já estáo muito ocupados com os problemas de saúde (n=64). \\
\hline \multirow{2}{*}{ Respostas } & Antes & Depois & Teste McNemar-Bowker \\
& n (\%) & n (\%) & \\
\hline Discordam & $46(71,9)$ & $30(46,9)$ & $\chi^{2}=13,95 ; \mathrm{p}=0,003$ \\
Neutros & $07(10,9)$ & $21(32,8)$ & \\
Concordam & $11(17,2)$ & $13(20,3)$ & \\
\hline
\end{tabular}

Tabela 3. Interesse profissional sobre o padräo da violência doméstica, os riscos de saúde relacionados e o aconselhamento para diminuir ou pará-la.

C) Eu náo sei como eu abordaria efetivamente os pacientes para reduzir a violência doméstica (n=65).

\begin{tabular}{lccc}
\hline Respostas & $\begin{array}{c}\text { Antes } \\
\mathbf{n}(\%)\end{array}$ & $\begin{array}{c}\text { Depois } \\
\mathbf{n}(\%)\end{array}$ & Teste McNemar-Bowker \\
\hline Discordam & $15(23,1)$ & $21(32,3)$ & \\
Neutro & $19(29,2)$ & $24(36,9)$ & $\chi^{2}=8,37 ; \mathrm{p}=0,04$ \\
Concordam & $31(47,7)$ & $20(30,8)$ & \\
\hline
\end{tabular}

E) Náo há tempo suficiente para aconselhar os pacientes sobre a violência doméstica $(\mathrm{n}=62)$.

\begin{tabular}{lccc}
\hline Respostas & $\begin{array}{c}\text { Antes } \\
\text { n (\%) }\end{array}$ & $\begin{array}{c}\text { Depois } \\
\text { n (\%) }\end{array}$ & Teste McNemar-Bowker \\
\hline Discordam & $40(64,5)$ & $32(51,6)$ & \\
Neutro & $7(11,3)$ & $17(27,4)$ & $\chi^{2}=7,68 ; \mathrm{p}=0,05$ \\
Concordam & $15(24,2)$ & $13(21,0)$ & \\
\hline
\end{tabular}


para realizar. Em contrapartida, fazer perguntas usando um questionário de detecção de violência doméstica foi o item no qual os respondentes apontaram ter maior confiança para executar - $16(24,6 \%)$ participantes responderam ter nenhuma ou pouca confiança e 49 $(75,4 \%)$, pouca ou muita confiança.

\section{Discussão}

Os resultados demonstraram que, no que diz respeito a lidar com vítimas de violência doméstica, os profissionais têm mais conhecimento sobre as causas e o aconselhamento às vítimas, com participação na capacitação. Ao passo que diminuiu o número de profissionais que acreditavam não terem muito a oferecer aos pacientes em situaçáo de violência doméstica. Nesse sentido, o conteúdo e a forma de apresentação da capacitação parecem ter sensibilizado os profissionais sobre aspectos teóricos relevantes, bem como a importância de sua contribuição no atendimento a pacientes envolvidos neste tipo de situação.

Esses dados convergem com estudos que indicaram que vários profissionais acreditam não haver nada a ser feito, atribuindo à falta de credibilidade que têm em outros setores. Essa crença contribui para a falta de comprometimento em relação à detecção e ao encaminhamento, bem como ao trabalho em rede. Além disso, tais estudos apontam barreiras pessoais dos profissionais como fator que desfavorece a implementação de açôes adequadas para enfrentar a violência doméstica nos serviços de saúde. Destacam-se, entre elas, o sentimento de impotência frente à situação, bem como o receio da reação do agressor diante de alguma intervenção (Rodríguez-Bolaños, MárquezSerrano \& Kageyama-Escobar, 2005; Kiss, 2004; De Ferrante, Santos \& Vieira, 2009). Isso corrobora também com, um estudo encontrado sobre crenças dos profissionais de saúde, realizado em município da mesma regiáo da presente pesquisa, no qual foi apontado que em relação às providências tomadas diante da suspeita ou confirmação do ato violento, os agentes comunitários de saúde admitiram não tomar nenhuma nos casos de violência doméstica, apresentando como principais entraves a falta de apoio e o acompanhamento dos órgãos jurídicos e o medo denunciar, justificado pelo possível comprometimento na comunidade onde vivem e trabalham (Lourenço et al., 2010).

Segundo Moura e Reichenheim (2005), a violência doméstica não é identificada nas abordagens rotineiras dos serviços de saúde, por estas serem baseadas em práticas curativas que se fundamentam na observaçáo de sinais e sintomas. Dessa forma, a violência, manifestada de forma mais clara em casos mais agressivos, pode mais facilmente ser detectada pelo profissional, o que não ocorre em casos menos evidentes e que podem se tornar mais graves no futuro. Os treinamentos e as capacitaçóes que visem desenvolver a habilidade do profissional para realizar açôes de prevenção à violência doméstica devem, portanto, enfatizar a importância do mesmo em se interessar em abordar questóes que apontem sinais da existência de possíveis relações violentas, independente da observação de agressóes claras. Partindo para a possibilidade de se intervir antes que estas ocorram.

Pesquisasobrepráticasdosprofissionais dasequipes de saúde da família voltadas para mulheres em situação de violência sexual aponta a necessidade de discutila na rotina dos serviços, capacitando os profissionais e realizando um trabalho com outras instituições e setores envolvidos (Oliveira, 2005). Tal fato também foi apontado em um estudo realizado com profissionais de saúde, em relação a suas crenças sobre violência contra crianças e adolescentes (Gebara \& Lourenço, 2008). Nesse, a maior parte dos profissionais afirmou nunca ter recebido nenhum tipo de capacitação, deixando clara a inexistência de uma política de treinamento científico para a violência doméstica no local pesquisado.

Algumas razões são apontadas pela literatura para a baixa identificação de casos de violência intrafamiliar ou doméstica, entre elas a formação e atuação do profissional de saúde, que deve estar sensibilizado ao problema, tendo em vista que muitos casos náo se apresentam de forma clara. Além disso, também podem ser citados: a percepção de que o tempo é insuficiente para o atendimento, a crença de que não existem recursos disponíveis para oferecer às vítimas, o receio em abordar o assunto, $\mathrm{o}$ ato de acreditar que isso náo faz parte de sua função e o sentimento de impotência frente às situaçôes (Moura \& Reichenheim, 2005).

Quanto ao interesse do profissional, os resultados indicaram que a capacitaçáo pode ter contribuído de alguma forma para que o profissional se sentisse apto a abordar os pacientes em relaçáo à violência doméstica, tendo em vista o decréscimo de profissionais que concordavam com a afirmação de que não sabem abordar os pacientes quanto à questáo. Por outro lado, pode esclarecer melhor a respeito de como vítimas podem ser auxiliadas antes de apresentarem claramente agressóes.

Os resultados indicaram que, após a capacitação, tanto os profissionais que concordavam, como aqueles 
que discordavam, passaram a se posicionar de forma neutra em relaçáo à afirmativa de que não há tempo suficiente para aconselhar pacientes sobre violência doméstica. Destaca-se a necessidade de maior esclarecimento de que o aconselhamento pode ser realizado de forma efetiva e breve e que esta ação pode favorecer uma melhora da saúde global do paciente, aprimorando uma atuação preventiva e de promoção de saúde. Apesar da mudança descrita, esta não foi uma crença presente para a maioria dos profissionais. Esse dado vai de encontro com resultados de outros estudos, que apontam a ideia de que não existe tempo suficiente para tratar da questáo nos atendimentos (Moura \& Reichenheim, 2005, Moreira et al., 2008).

Em relação à confiança que os profissionais de saúde sentem em sua habilidade para lidar com casos de violência doméstica, os resultados não apontam mudanças significativas quando são comparados os dados coletados antes e após a capacitação. Vale enfatizar que a proposta do projeto de disseminação das práticas de prevenção, no qual se insere este estudo, era introduzir a temática da violência doméstica, com o intuito de sensibilizar o profissional de saúde de que a temática está intimamente relacionada à sua prática, e sua atuação poderia favorecer significativamente a prevençáo, tanto no que tange ao agravamento de casos existentes, quanto ao aparecimento de novos.

Tendo isso em vista, esta pesquisa não foi suficiente para desenvolver o sentimento de confiança nos profissionais, o que, talvez, demandasse um maior aprofundamento do conteúdo apresentado, bem como uma atuação de mobilizaçáo da rede dos municípios que integraram o projeto. A complexidade do fenômeno exige o envolvimento de outros setores para o desenvolvimento de soluçôes integradas (Malta et al., 2007; Andrade \& Fonseca, 2008).

Segundo recomendação feita no Manual de Recursos e Estratégias de Combate à Violência Doméstica da Organização das Nações Unidas (2003), somente com informação precisa será possível promover formação adequada para dar seguimento ao desenvolvimento de propostas efetivas de combate à violência. $\mathrm{O}$ conhecimento e a credibilidade, na rede de enfrentamento à violência doméstica, por parte do profissional de saúde, podem contribuir significativamente para que o mesmo se interesse e se sinta confiante em realizar açôes nesse sentido. Destaca-se a fragilidade da rede dos municípios em que este estudo foi realizado, o que pode refletir na confiança que o profissional sente para realizar açóes preventivas propostas na capacitaçáo realizada, tal como detectar casos de forma rotineira nos atendimentos.

Os resultados de um estudo apontam as limitaçôes dos profissionais para identificar possíveis casos de violência (Moreira et al., 2008), no qual os profissionais entrevistados apresentaram dificuldades no que se refere à identificação, ao atendimento e ao encaminhamento de mulheres em situação de violência para os serviços especializados, o que reforça a ideia por parte dos profissionais de que estes não estão capacitados o suficiente para lidar com este problema.

Aponta-secomolimitação deste estudoo tamanho da amostra, sendo relevante enquanto uma experiência piloto, que inseriu a temática da violência doméstica em projeto de disseminaçáo das práticas de prevenção ao uso de substâncias psicoativas. Sáo necessárias futuras pesquisas que permitam maior extrapolação dos resultados, bem como a investigaçáo de crenças dos profissionais da atenção primária à saúde em relação à violência doméstica, visando analisar a associação entre a modificação de tais crenças e mudanças em termos de realização de intervençôes preventivas por parte dos profissionais em questão. Sugere-se também, a partir dos resultados observados, a avaliaçáo de impacto dos desdobramentos mencionados, de elaboração e distribuição de material didático e uma intervenção mais voltada para instrumentalizar os profissionais no rastreio da violência doméstica.

\section{Referências}

Andrade, C. J. M. \& Fonseca, R. M. G. S. (2008). Considerações sobre violência doméstica, gênero e o trabalho das equipes de saúde da família. Revista da Escola de Enfermagem, 42, 591-595.

Bhona, F. C., Lourenço, L. M. \& Brum, C. R. S. (2011). Violência Doméstica: um estudo bibliométrico. Arquivos Brasileiros de Psicologia, Rio de Janeiro, 63, 1-110.

Brasil. Ministério da Saúde. Secretaria de Políticas de Saúde. Departamento de Atenção Básica. (2002a). Avaliação da Implementação do Programa Saúde da Família em Dez Grandes Centros Urbanos sintese dos principais resultados. Brasília: Ministério da Saúde. Brasil. Ministério da Saúde. Secretaria de Políticas de Saúde. (2002b). Violência intrafamiliar: orientaçôes para prática em serviço. Cadernos de Atenção Básica No 8. Série A - Normas e Manuais Técnicos; $n^{\circ}$ 131. Brasília: Ministério da Saúde. 
Cavazza, N. (2008). Psicologia das atitudes e das opinióes. São Paulo: Ediçóes Loyola.

De Ferrante, F. G., Santos, M. A. \& Vieira, E. M. (2009). Violência contra a mulher: percepçáo dos médicos das unidades básicas de saúde da cidade de Ribeirão Preto, São Paulo. Interface Comunicação Saúde Educação, 13, 287-299.

Ellsberg, M., Jansen, H. A. F. M., Heise, L., Watts, C. H. \& Garcia-Moreno, C. (2008). Intimate partner violence and women's physical and mental health in the WHO multi-country study on women's health and domestic and domestic violence: an observational study. Lancet, 371, 1165-1172.

Garcia-Moreno, C., Jansen, H. A. F. M., Ellsberg, M., Heise, L. \& Watts, C. H. (2006). Prevalence of intimate partner violence: findings from the WHO multi-country study on women's health and domestic violence. Lancet, 368, 1260-1269.

Gebara, C. F. P. \& Lourenço, L. M. (2008). Crenças dos Profissionais de Saúde sobre Violência Doméstica contra Crianças e Adolescentes. Psicologia em Pesquisa, 2, 27-39.

Gebara, C. F. P., Cezario, A. C. F., Ronzani, T. M. \& Lourenço, L. M. (2010). Violência Doméstica Praticada Contra Crianças e Adolescentes Segundo Crenças de Profissionais da Saúde. Actas do VII Simpósio Nacional de Investigação em Psicologia, Universidade do Minho, Portugal.

Kiss, L. B. (2004). Temas médico-sociais e a intervenção em saúde: a violência contra mulheres no discurso dos profissionais (Dissertação de Mestrado). São Paulo: Faculdade de Medicina, Universidade de São Paulo.

Krüger, H. R. (1995). Psicologia das Crenças: Perspectivas Teóricas. Tese (Concurso para professor titular de Psicologia Social). Rio de Janeiro: Universidade Federal do Rio de Janeiro.

Lourenço, L. M., Brum, C. R. S., Cruvinel, E., Gebara, C. F. P., Almeida, A. A., Cezario, A. C. F., Basílio, C. \& Senra, L. X. (2010). Violência Doméstica: O que é. O que fazer. Como previnir. Desenvolvimento de material didático ou instrucional - Folder.

Malta, D. C., Lemos, M. S. A., Silva, M. M. A., Rodrigues, E. M. S., Gazal-Carvalho, C. \& Neto, O. L. M. (2007). Iniciativas de vigilância e prevençáo de acidentes e violência no contexto do Sistema Único de Saúde (SUS). Epidemiologia e Serviços de Saúde, 16, 45-55.
Moreira, S. N. T., Galvão, L. L. L. F., Melo, C. O. M. \& Azevedo, G. D. (2008). Violência física contra a mulher na perspectiva de profissionais de saúde. Revista de Saúde Pública, 42, 1053-1059.

Moura, A. T. M. S. \& Reichenheim, M. E. (2005). Estamos realmente detectando violência familiar contra a criança em serviços de saúde? A experiência de um serviço público do Rio de Janeiro, Brasil. Cadernos de Saúde Pública, Rio de Janeiro, 21, 1124-1133.

Oliveira, C. C. (2005). Práticas dos profissionais de saúde da familia voltadas para mulheres em situaçâa de violência sexual: uma abordagem de gênero (Tese). São Paulo: Escola de Enfermagem, Universidade de São Paulo.

Organização das Naçóes Unidas. (2003). Centro para o Desenvolvimento Social e Assuntos Humanitários - Gabinete das Naçóes Unidas de Viena. Estratégias de combate à violência doméstica: manual de recursos/ONU. 128p. (E. F. G. Barros, Trad.). Lisboa: Direcção-Geral da Saúde.

Organização Mundial de Saúde. (2002). Relatório mundial sobre violência e saúde. Brasília: OMS/ OPAS.

Rodríguez-Bolaños, R. A., Márquez-Serrano, M. \& Kageyama-Escobar, M. L. (2005). Violencia de género: actitud y conocimiento del personal de salud de Nicaragua. Salud Publica Mexico, 47, 134-144.

Ronzani, T. M., Amaral, M. B., Souza-Formigoni, M. L. O. \& Babor, T. F. (2008). Evaluation of a training program to implement alcohol screening, brief intervention and referral to treatment in primary health care in Minas Gerais, Brazil. Nordic Studies on Alcohol and Drugs, 25, 529-538.

Ronzani, T. M., Mota, D. C. B. \& Souza, I. C. W. (2009). Prevenção do uso de álcool na atenção primária em municípios do estado de Minas Gerais. Revista de Saúde Pública, 43, 51-61.

Schraiber, L. B., D’Oliveira, A. F. P. L., Hanada, H., Figueiredo, W., Couto, M., Kiss, L., Durand, J. \& Pinho, A. (2003). Violência vivida: a dor que não tem nome. Interface (Botucatu), 7, 41-54.

Schraiber, L. B., D’Oliveira, A. F. P. L., França Jr, I., Diniz, S., Portella, A. P., Ludermir, A. B., Valença, O. \& Couto, M. T. (2007). Prevalência da violência contra a mulher por parceiro íntimo em regiōes do Brasil. Revista de Saúde Pública, 41, 797-807. 
Sinclair, D. (2010). Introdução à Violência Contra a Mulher. In L. C. A., Williams, J. M. D., Maia, \& K. S. A., Rios, (orgs), Aspectos Psicológicos da Violência: pesquisa e intervençâo cognitivocomportamental (pp. 69-83). Santo André: ESETec.

UN Millennium Project. (2005). Taking Action: Achieving Gender Equality and Empowering Women. Task Force on Education and Gender Equality. EARTHSCAN. London, Va.

Vieira, E. M., Perdona, G. S. C. \& Santos, M. A. (2011). Fatores associados à violência física por parceiro íntimo em usuárias de serviços de saúde. Revista de Saúde Pública, 45, 730-737.

\section{Endereço para correspondência:}

Camila Resende Soares Brum

Avenida Marechal Deodoro, 230, $5^{\circ}$ andar - Centro CEP 36013-000 - Juiz de Fora/MG

E-mail: resendecamila@gmail.com

Recebido em 21/09/2012

Revisto em 22/11/2012

Aceito em 16/05/2013

* Pesquisa financiada pela Fundação de Amparo à Pesquisa do Estado de Minas Gerais.

Agradecimentos: Fundaçáo de Amparo à Pesquisa do Estado de Minas Gerais, colaboradores e bolsistas de iniciação científica do Polo de Psicologia Social e Saúde Coletiva que participaram do projeto: Leonardo Fernandes Martins, Erica Cruvinel, Daniela Cristina Belchior Mota, Ana Cláudia Ferreira Cezário, Marina de Castro Oliveira, Tamires Jordão Laport e Tassiana Cristina Mendes Miranda. 